\title{
Influencia de la adolescencia y su entorno en la adherencia al control prenatal e impacto sobre la prematuridad, bajo peso al nacer y mortalidad neonatal
}

\author{
Luis Alfonso Mendoza Tascón ${ }^{1,2}$, Martha Deyfilia Arias Guatibonza ${ }^{2}$, Claudia Bibiana \\ Peñaranda Ospina ${ }^{2}$, Laura Isabel Mendoza Tascón ${ }^{1}$, Stefania Manzano Penagos ${ }^{1}$, Ana \\ María Varela Bahena. 1 \\ ${ }^{1}$ Programa de Medicina, Facultad de Ciencias de la Salud, Unidad Central del Valle. ${ }^{2}$ Fundación Hospital San José de \\ Buga. Colombia.
}

Fuente de financiamiento: La Universidad Central del Valle aportó recursos económicos para la recolección de la información y digitación de la base de datos.

\section{RESUMEN}

Antecedentes: La morbimortalidad materna y neonatal está incrementada en adolescentes y gestantes sin control prenatal o con una mala adherencia. Objetivo: Identificar la influencia de la adolescencia y su entorno sobre la adherencia al control prenatal y su impacto sobre la prematuridad y/o bajo peso al nacer y la mortalidad neonatal. Métodos: Estudio de casos y controles, realizado con 570 adolescentes y 2093 gestantes $\geq 20$ años de edad, cuyos neonatos fueron hospitalizados en una unidad neonatal. Las asociaciones se determinaron mediante Odds Ratio, con su intervalo de confianza de 95\%, empleándose la regresión logística en el análisis multivariado. Para la construcción del Modelo con Árboles de Clasificación y Regresión se emplearon variables que en el bivariado hubieran tenido un valor $p<0,05$. Resultados: La edad materna 13-19 años se halló asociada a mala adherencia al control prenatal, después de ajustarse por escolaridad $<11$ años, carencia de esposo o compañero permanente, carencia de seguridad social en salud y antecedente de gravidez $\geq 2$ gestaciones. Esta mala adherencia al control prenatal se asoció con aumento de riesgo de prematuridad y/o bajo peso al nacer y de la mortalidad neonatal. Conclusiones: La adolescencia se halló independientemente asociada a una mala adherencia al control prenatal, sin embargo, existen otros determinantes importantes de esta mala adherencia, que a su vez se halló asociada con prematuridad y/o bajo peso al nacer, y con mortalidad neonatal.

\section{PALABRAS CLAVE: Adolescencia, control prenatal, prematuridad, bajo peso al nacer, mortalidad neonatal}

\section{SUMMARY}

Background: Maternal morbidity and neonatal mortality is increased in adolescents and pregnant women without prenatal care or poor adherence. Objective: To identify the influence of adolescence and its surroundings on adherence to prenatal care and its impact on prematurity and/or low birth weight and neonatal mortality. Methods: Case-control study, conducted with 570 pregnant adolescents and $2093 \geq 20$ years of age whose infants were hospitalized in a neonatal unit. Associations were determined by Odds Ratio with confidence interval of $95 \%$, using the multivariate logistic regression analysis. To build the model with Classification and Regression Trees variables in the bivariate had had a $p<0.05$ was used. Results: Maternal age 13-19 was found associated with poor adherence to prenatal care, after adjusting for schooling $<11$ 
years, lack of spouse or life partner, lack of social security in health and pregnancy history of $\geq 2$ pregnancies. This poor adherence to prenatal care was associated with increased risk of prematurity and/or low birth weight and increased neonatal mortality. Conclusions: The teen was found independently associated with poor adherence to prenatal care, but there are other important determinants of poor adherence, which in turn was found associated with prematurity and / or low birth weight, and neonatal mortality.

\section{KEY WORDS: Adolescence, prenatal care, prematurity, low birth weight, neonatal mortality}

\section{INTRODUCCIÓN}

La adolescencia es una etapa en la cual el niño/a alcanza su madurez física, psicológica, afectiva, intelectual y social (1), y es un momento de transformación, vulnerabilidad y oportunidades. Los y las adolescentes se definen como individuos entre las edades de 10 y 19 años, según la Organización Mundial de la Salud (OMS) (2).

La actividad sexual (AS) cada vez ocurre a más temprana edad (promedios de inicio 13,5-16 años) $(3,4)$, mientras el embarazo en la adolescencia se constituye en un problema de origen multifactorial, con mayor riesgo de complicaciones para la madre, feto y neonato (5). En los países en desarrollo, entre $15-20 \%$ de todos los nacimientos corresponden a madres adolescentes (6) y $40 \%$ de estos embarazos no son deseados (7). Cerca de 16 millones de adolescentes entre 15-19 años tienen un hijo cada año. Colombia registra una de las mayores tasas de embarazo adolescente de América Latina, donde 1 de cada 5 adolescentes entre 15-19 años ha estado embarazada $(8,9)$. Holguin y cols (10) en una ciudad colombiana, encontraron que $7,2 \%$ de las adolescentes escolarizadas afirmaron haber estado embarazada alguna vez.

La evidencia hasta la fecha sugiere que la morbilidad y mortalidad materna se incrementan durante el embarazo adolescente $(6,11-13)$, especialmente entre las más jóvenes (10-14 años) (14,15). Para la Organización Panamericana de la Salud (OPS) el $95 \%$ de muertes maternas son evitables y es posible disminuir esa cifra a menos de 10 muertes por cada $100.000 \mathrm{nv}$, con un adecuado y oportuno programa de control prenatal (CPN) y atención del parto. El CPN permite disminuir la morbi-mortalidad maternoperinatal, debido a la identificación oportuna, la prevención y manejo de factores de riesgo (16). El CPN tiene como objetivo la prevención y reducción de la mortalidad materna y morbi-mortalidad perinatal y está encaminado a promover la salud del binomio madre-hijo y obtener finalmente un neonato sano (17). La OMS considera que un CPN de buena calidad es aquel que inicia en el primer trimestre del embarazo, donde se eduque, proteja, detecte y haga tratamiento de enfermedades que puedan aparecer en la gestación, con al menos 5 citas, idealmente
8, durante el embarazo donde el médico realice la primera y participe en las siguientes citas (18). Para Colombia, Resolución 412 de año 2000, entre sus objetivos está desarrollar acciones que identifiquen oportuna y efectivamente las enfermedades del binomio madre-hijo, realizando gestión y manejo de riesgo. Estas acciones se desarrollan dentro del Programa de Detección Temprana de Alteraciones del Embarazo, que en los prestadores de los servicios de salud se denomina CPN (18).

El objetivo del estudio fue identificar la influencia de la adolescencia y su entorno, sobre la adherencia al control prenatal y su impacto sobre la prematuridad y/o bajo peso al nacer y la mortalidad neonatal.

\section{SUJETOS Y MÉTODOS}

Diseño del estudio: La información para esta investigación fue recolectada como parte de un estudio observacional, de tipo casos y controles, realizado en la Unidad de Cuidados Intensivos Neonatales de la Fundación Hospital San José de Buga, Colombia, entre madres adolescentes, jóvenes y adultas y sus neonatos, que fueron hospitalizados entre septiembre de 2005 y diciembre de 2014.

Población: Se incluyeron madres adolescentes (13-19 años), jóvenes (20-25 años) y adultas (2635 años) y sus hijos con peso nacer $\geq 500$ gramos $y \geq 24$ semanas de edad gestacional, cuya permanencia haya sido hospitalización por enfermedad y no por falta de acudiente, excluyéndose gestantes y neonatos con datos importantes incompletos en las historias clínicas.

Fuente de los datos: Los datos fueron obtenidos de historias clínicas maternas y neonatales, obtenidas del archivo de la Fundación Hospital San José de Buga, Colombia.

Variables dependientes: La variable de resultado primaria para este trabajo fue la mala adherencia al control prenatal, definido como el inicio del CPN después de las doce semanas de gestación y un número de visitas durante este CPN inferior a cinco; se evaluó el impacto de la mala adherencia al CPN sobre el nacimiento prematuro y/o con bajo peso al nacer, definidos como nacimiento antes de las 37 semanas de edad gestacional determinado 
por la Calificación de Ballard Modificado y peso al nacer inferior a 2500 g; además, se evaluó el impacto de la mala adherencia al CPN sobre la mortalidad neonatal.

Variables independientes: Para el resultado primario, mala adherencia al CPN, la variable independiente de interés fue el grupo de edad, comparando las mujeres con edades entre 13-19 años y mujeres entre 20-35 años. Se tuvieron en cuenta las covariables escolaridad, estado civil, carencia de seguridad social en salud y gravidez. Para evaluar el impacto sobre el nacimiento prematuro y/o con bajo peso al nacer, la variable de interés tenida en cuenta fue mala adherencia al CPN y las covariables edad materna, escolaridad, estado civil, carencia de seguridad social en salud, gravidez y complicaciones maternas (ruptura prematura de membrana ovulares, infección de vías urinaria materna, trabajo de parto prematuro, preeclampsia y corioamnionitis). Para el impacto muerte neonatal, la variable de interés tenida en cuenta fue mala adherencia al CPN y covariables como edad materna, escolaridad, estado civil, carencia de seguridad social en salud, gravidez, prematuridad y/o bajo peso al nacer y complicaciones maternas (ruptura prematura de membrana ovulares, infección de vías urinaria materna, trabajo de parto prematuro, preeclampsia y corioamnionitis). Otras variables evaluadas incluyó edad gestacional y peso al nacer, vía de nacimiento y estancia neonatal.

Limitaciones y sesgos: Los principales sesgos tenidos en cuenta fueron los de información, al tratarse de estudio en registros de historias clínicas. Para prevenirlos, se excluyeron aquellos registros que carecieran de datos importantes tanto de las variables independientes, como de las dependientes.

Tamaño de la muestra y muestreo: Ingresaron al estudio todas las gestantes y sus hijos que cumplieran con los criterios de inclusión y carecieran de criterios de exclusión, que fueron 570 madres adolescentes y sus hijos y 2093 mujeres jóvenes y adultas y sus hijos. Este tamaño de muestra con un nivel de significancia de $95 \%$ y un poder estadístico de $80 \%$, nos permitió calcular un OR de 1,37.

Análisis estadístico: Los datos fueron trasladados a Stata® versión 11.0 para su análisis. Las variables cuantitativas se expresaron como promedios y desviación estándar (DE) o medianas y rangos intercuartiles $(\mathrm{RI})$ y se evaluaran con la pruebas t no pareado o Mann-Whitney, de acuerdo a su distribución para comparar diferencias. Las variables categóricas se expresaron en frecuencias y proporciones y fueron comparadas usando la pruebas Chi2 o Exacta de Fisher. Para determinar la asociación entre variables independientes y de resultado, se calculó el Odds Ratio (OR) con sus respectivos intervalos de confianza del 95\% (IC 95\%). El análisis multivariado se realizó mediante regresión logística $(R L)$. Todas las pruebas fueron de dos colas y se consideró un valor de $p<0,05$ como estadísticamente significativo. Para el análisis con Árboles de Clasificación y Regresión se empleó el módulo Answer Tree del sofware SPSS $®$ 19.0, mediante el método de crecimiento CART construyendo el árbol para una mala adherencia al CPN con variables que en el bivariado hayan tenido una $p<0,05$, utilizando validación cruzada y la medición de la pureza del modelo se realizó empleando Gini con un cambio mínimo de mejora 0,0001.

Consideraciones éticas: La investigación fue aprobada por el Comité de Ética institucional y careció de conflicto de intereses.

\section{RESULTADOS}

Determinantes demográficos. Se incluyeron 570 adolescentes entre 13-19 años, 755 jóvenes entre 20-25 años y 1338 adultas entre 26-35 años. Se excluyeron 669 madres y sus hijos por ausencia de datos importantes en los registros hospitalarios. La Tabla I muestra las principales características maternas, donde identificamos diferencias estadísticamente significativas $(p<0,0001)$ entre adolescentes y mujeres adultas, como menor escolaridad, menor proporción de mujeres casadas o que viven en unión libre y mayor carencia de seguridad social en salud para las adolescentes.

Determinantes gestacionales, del cuidado prenatal y neonatales. La Tabla II resume las diferencias de los determinantes gestacionales, del cuidado prenatal y neonatales para adolescentes y mujeres adultas. En esta resaltamos, una significativa menor gravidez, mayor edad gestacional de inicio del CPN, menor número de visitas a CPN y mayor proporción de nacimientos por cesárea entre las madres adolescentes $(p<0,0001)$. También identificamos una significativa mayor proporción de preeclampsia $(p=0,0073)$ y corioamnionitis $(p=0,0450)$ entre las mujeres adultas. No hallamos diferencias significativas para otras complicaciones gestacionales (ruptura prematura de membranas, infección de vías urinarias maternas y trabajo de parto prematuro), como tampoco para edad gestacional y peso al nacer, estancia neonatal hospitalaria, ni mortalidad neonatal. 


\section{DETERMINANTES MATERNOS SEGÚN LA EDAD}

\begin{tabular}{|c|c|c|c|c|}
\hline Determinantes & & $\begin{array}{l}\text { Edad } 13-19 \text { años } \\
\text { n: } 570\end{array}$ & $\begin{array}{l}\text { Edad: } 20-35 \text { años } \\
\text { n: } 2093\end{array}$ & $\mathrm{p}$ \\
\hline \multirow[t]{6}{*}{ Edad materna (años) } & & $17(16-19)$ & $27(23-31)$ & $<0,0001$ \\
\hline & 13-14 años & $21(3,7 \%)$ & & \\
\hline & $15-16$ años & $150(26,3 \%)$ & & \\
\hline & 17-19 años & $399(70 \%)$ & & \\
\hline & 20-25 años & & $755(36,1 \%)$ & \\
\hline & $26-35$ años & & $1338(63,9 \%)$ & \\
\hline \multirow[t]{4}{*}{ Escolaridad en años de estudio } & & $9(6-11)$ & $11(8-11)$ & $<0,0001$ \\
\hline & 0-5 años de escolaridad & $103(18,1 \%)$ & $365(17,4 \%)$ & \\
\hline & 6-10 años de escolaridad & $280(49,1 \%)$ & $338(16,1 \%)$ & \\
\hline & 11 años de escolaridad & $175(30,7 \%)$ & $1183(56,5 \%)$ & \\
\hline \multirow{4}{*}{$\begin{array}{l}\text { Más de } 11 \text { años de escolaridad } \\
\text { Estado civil }\end{array}$} & & $12(2,1 \%)$ & $207(9,9 \%)$ & $<0,0001$ \\
\hline & & & & $<0,0001$ \\
\hline & Casada o unión libre & $421(73,9 \%)$ & $1824(87,3 \%)$ & \\
\hline & Soltera, separada, viuda & $149(26,1 \%)$ & $266(12,7 \%)$ & \\
\hline $\begin{array}{l}\text { Carencia de seguridad social } \\
\text { en salud }\end{array}$ & & $71(12,4 \%)$ & $83(4 \%)$ & $<0,0001$ \\
\hline
\end{tabular}

Tabla II

DETERMINANTES GESTACIONALES, DEL CUIDADO PRENATAL Y NEONATALES SEGÚN LA EDAD

\begin{tabular}{|c|c|c|c|c|}
\hline \multicolumn{2}{|l|}{ Determinantes } & $\begin{array}{l}\text { Edad 13-19 años } \\
\text { n: } 570\end{array}$ & $\begin{array}{c}\text { Edad: } 20-35 \text { años } \\
\text { n: } 2093\end{array}$ & $\mathrm{p}$ \\
\hline \multicolumn{2}{|c|}{ Gravidez incluido neonato del estudio } & $1(1-1)$ & $2(1-3)$ & $<0,0001$ \\
\hline \multicolumn{2}{|c|}{ Edad gestacional del inicio de CPN } & $10(7-16)$ & $8(4-12)$ & $<0,0001$ \\
\hline \multicolumn{2}{|c|}{ Edad gestacional del inicio de CPN >12 semana } & $185(32,5 \%)$ & $353(16,9 \%)$ & $<0,0001$ \\
\hline \multicolumn{2}{|l|}{ Número de visitas en CPN } & $6(4-8)$ & $7(5-8)$ & $<0,0001$ \\
\hline \multicolumn{2}{|l|}{ Número de visitas en $\mathrm{CPN}<5$} & $152(26,7 \%)$ & $350(16,7 \%)$ & $<0,0001$ \\
\hline \multicolumn{2}{|c|}{ Mala adherencia al CPN (inicio de CPN $>12$ semanas $\mathrm{y}<5$ visitas) } & $195(34,2 \%)$ & $434(20,7 \%)$ & $<0,0001$ \\
\hline \multirow[t]{5}{*}{ Complicaciones obstétricas: } & Ruptura prematura de membranas & $69(12,1 \%)$ & $244(11,7 \%)$ & 0,7688 \\
\hline & Infección de vías urinarias & $92(16,1 \%)$ & $312(14,9 \%)$ & 0,4450 \\
\hline & Trabajo de parto prematuro & $2(0,35 \%)$ & $6(0,29 \%)$ & 0,8040 \\
\hline & Preeclampsia & $47(8,2 \%)$ & $257(12,3 \%)$ & 0,0073 \\
\hline & Corioamnionitis & $22(3,9 \%)$ & $49(2,3 \%)$ & 0,0450 \\
\hline \multicolumn{2}{|l|}{ Nacimiento por cesárea } & $176(30,9 \%)$ & $269(12,9 \%)$ & $<0,0001$ \\
\hline \multicolumn{2}{|c|}{ Edad gestacional al nacer (semanas) } & $38(35-39)$ & $38(35-39)$ & 0,8846 \\
\hline \multicolumn{2}{|c|}{ Edad gestacional al nacer $<37$ semanas } & $197(34,6 \%)$ & $722(34,5)$ & 0,9830 \\
\hline \multicolumn{2}{|l|}{ Peso al nacer (gramos) } & $2835(2270-3300)$ & $2920(2300-3325)$ & 0,1163 \\
\hline \multicolumn{2}{|l|}{ Peso al nacer $<2500 \mathrm{~g}$} & $188(33 \%)$ & $653(31,2 \%)$ & 0,4170 \\
\hline \multicolumn{2}{|c|}{ EG al nacer $<37$ semanas $y / o$ peso al nacer $<2500 \mathrm{~g}$} & $225(39,5 \%)$ & $803(38,4 \%)$ & 0,6300 \\
\hline \multicolumn{2}{|l|}{ Estancia hospitalaria en días } & $4(3-8)$ & $4(2-7)$ & 0,0906 \\
\hline \multicolumn{2}{|l|}{ Muertes } & $9(1,6 \%)$ & $31(1,5 \%)$ & 0,8650 \\
\hline
\end{tabular}

CPN: control prenatal. 
Determinantes de mala adherencia al control prenatal e impacto sobre el nacimiento prematuro y/o con bajo peso al nacer y sobre la mortalidad neonatal. La Tabla III muestra la mala adherencia al CPN que tuvieron las adolescentes, además de la escolaridad $<11$ años, el estado civil soltera, separada o viuda, carencia de seguridad social en salud y antecedente de gravidez $\geq 2$ gestaciones. La Tabla IV, muestra el impacto negativo que tiene la mala adherencia al CPN y otros factores como edad materna entre 13-15 años, escolaridad $<11$ años, gravidez $\geq 2$ gestaciones, preeclampsia y corioamnionitis sobre el parto prematuro y/o bajo peso al nacer. Por otro lado, como mostramos en la Tabla $\mathrm{V}$, la mala adherencia al CPN también tiene un impacto negativo sobre la mortalidad neonatal, con una mayor proporción de muertes entre aquellas madres no adherentes a este, así como el impacto negativo sobre las muertes neonatales de otros determinantes como, carencia de seguridad social en salud, nacimiento prematuro y/o con bajo peso al nacer.

La Tabla VI identifica en el análisis bivariado y multivariado los determinantes para una mala adherencia al CPN, siendo estos la adolescencia de 13-19 años, escolaridad $<11$ años, estado civil soltera, separada o viuda, carencia de seguridad social en salud y antecedente de gravidez $\geq 2$ gestaciones.

La Tabla VII en el análisis multivariado identifica como factores de riesgo para una mayor prematuridad y/o bajo peso al nacer a la mala adherencia al CPN, escolaridad $<11$ años, antecedente de gravidez $\geq 2$ gestaciones, preeclapmsia y corioamnionitis. La adolescencia no fue identificada como un factor de riesgo para estos eventos.

Tabla III

DETERMINANTES DE LA ADHERENCIA AL CONTROL PRENATAL

\begin{tabular}{lccc}
\hline Determinantes & $\begin{array}{c}\text { Mala adherencia al } \\
\text { CPN. n: 629 }\end{array}$ & $\begin{array}{c}\text { Buena adherencia al } \\
\text { CPN. n: 2034 }\end{array}$ & $p$ \\
\hline Adolescencia de 13 a 15 años & $32(5,1 \%)$ & $49(2,4 \%)$ & 0,0001 \\
Adolescencia de 16 a 19 años & $163(25,9 \%)$ & $434(21,3 \%)$ & $<0,0001$ \\
Mujeres de 20 a 35 años & $434(67 \%)$ & $1551(76,3 \%)$ & $<0,0001$ \\
Menos de 11 años de escolaridad & $316(50,2 \%)$ & $704(34,6 \%)$ & $<0,0001$ \\
Madre soltera, separada o viuda & $164(26 \%)$ & $252(12,4 \%)$ & $<0,0001$ \\
Carencia de seguridad social en salud & $79(12,6 \%)$ & $75(3,7 \%)$ & $<0,0001$ \\
Gravidez 2 y más & $381(60,6 \%)$ & $1078(53 \%)$ & 0,0010
\end{tabular}

CPN: control prenatal.

\section{Tabla IV}

DETERMINANTES QUE IMPACTAN SOBRE LA PREMATURIDAD Y/O BAJO PESO AL NACER

\begin{tabular}{lccc}
\hline Determinantes & $\begin{array}{c}\text { Prematuridad y/o bajo } \\
\text { peso al nacer. n: 629 }\end{array}$ & $\begin{array}{c}\text { Neonato de término y/o con peso } \\
\text { adecuado al nacer. n: 2034 }\end{array}$ & $\begin{array}{c}\mathrm{p} \\
\text { Adolescencia de 13 a 15 años }\end{array}$ \\
Adolescencia de 16 a 19 años & $40(3,9 \%)$ & $41(2,5 \%)$ & 0,0460 \\
Mujeres de 20 a 35 años & $185(18 \%)$ & $304(18,6 \%)$ & 0,8270 \\
Menos de 11 años de escolaridad & $803(78,1 \%)$ & $1290(78,9 \%)$ & 0,6300 \\
Madre soltera, separada o viuda & $448(44,4 \%)$ & $555(34,4 \%)$ & $<0,0010$ \\
Carencia de seguridad social en salud & $161(15,7 \%)$ & $254(15,5 \%)$ & 0,9324 \\
Mala adherencia al CPN & $65(6,3 \%)$ & $89(5,5 \%)$ & 0,3458 \\
Gravidez 2 y más & $294(28,6 \%)$ & $335(20,5 \%)$ & $<0,0010$ \\
Preeclampsia & $615(59,8 \%)$ & $844(51,6 \%)$ & $<0,0010$ \\
Corioamnionitis & $209(20,3 \%)$ & $92(5,6 \%)$ & $<0,0010$ \\
\end{tabular}

CPN: control prenatal. 
Tabla V

DETERMINANTES QUE IMPACTAN SOBRE MORTALIDAD NEONATAL

\begin{tabular}{lccc}
\hline Determinantes & $\begin{array}{c}\text { Egreso muerto } \\
\text { n: } 40\end{array}$ & $\begin{array}{c}\text { Egreso vivo } \\
\text { n: } 2623\end{array}$ & $\mathrm{p}$ \\
\hline Adolescencia de 13 a 15 años & $2(5 \%)$ & $79(3 \%)$ & 0,4755 \\
Adolescencia de 16 a 19 años & $7(17,5 \%)$ & $482(18,4 \%)$ & 0,9346 \\
Mujeres de 20 a 35 años & $31(77,5 \%)$ & $2062(78,6 \%)$ & 0,8648 \\
Menos de 11 años de escolaridad & $14(35 \%)$ & $989(38,3 \%)$ & 0,6697 \\
Madre soltera, separada o viuda & $8(20 \%)$ & $407(15,5 \%)$ & 0,4398 \\
Carencia de seguridad social en salud & $7(17,5 \%)$ & $147(5,6 \%)$ & 0,0014 \\
Mala adherencia al CPN & $21(52,5 \%)$ & $608(23,2 \%)$ & $<0,0010$ \\
Gravidez 2 y más & $25(62,5 \%)$ & $1434(54,7 \%)$ & 0,3298 \\
Prematuridad y/o bajo peso al nacer & $31(77,5 \%)$ & $997(38 \%)$ & $<0,0010$ \\
Preeclampsia & $8(20 \%)$ & $296(11,3 \%)$ & 0,0854 \\
Corioamnionitis & $1(2,5 \%)$ & $70(2,7 \%)$ & 0,9448 \\
\hline
\end{tabular}

CPN: control prenatal.

Tabla VI

DETERMINANTES DE LA ADHERENCIA AL CONTROL PRENATAL

\begin{tabular}{lcccc}
\hline \multirow{2}{*}{ Determinantes } & \multicolumn{3}{c}{ Mala adherencia al CPN } \\
\cline { 2 - 5 } & ORc (IC 95\%) & $\mathrm{p}$ & ORa (IC 95\%) & $\mathrm{p}$ \\
\hline Adolescencia de 13 a 19 años & $1,99(1,61-2,44)$ & $<0,0001$ & $1,85(1,44-2,39)$ & $<0,0001$ \\
Menos de 11 años de escolaridad & $1,91(1,58-2,30)$ & $<0,0001$ & $1,39(1,14-1,71)$ & $<0,0001$ \\
Madre soltera, separada o viuda & $2,47(2,66-5,30)$ & $<0,0001$ & $2,20(1,74-2,79)$ & $<0,0001$ \\
Carencia de seguridad social en salud & $3,76(2,66-5,30)$ & $<0,0001$ & $2,76(1,95-3,90)$ & $<0,0001$ \\
Gravidez 2 y más & $1,36(1,12-1,63)$ & $<0,001$ & $1,70(1,38-2,14)$ & $<0,0001$ \\
\hline
\end{tabular}

CPN: control prenatal.

Tabla VII

IMPACTO DE LA MALA ADHERENCIA AL CONTROL PRENATAL AJUSTADO POR OTRAS COVARIABLES SOBRE LA PREMATURIDAD Y/O BAJO PESO AL NACER

\begin{tabular}{lcccc}
\hline Determinantes & $\begin{array}{c}\text { Prematuridad } \\
\text { ORc (IC 95\%) }\end{array}$ & y/o & Bajo peso al nacer & $p$ \\
ORa (IC 95\%) & p & $1,66(1,33-2,06)$ & $<0,0001$ \\
Mala adherencia al CPN & $1,55(1,29-1,87)$ & $<0,0001$ & $1,33(0,80-2,22)$ & 0,2710 \\
Adolescencia de 13 a 19 años & $1,57(0,98-2,51)$ & 0,0459 & $1,50(1,22-1,84)$ & $<0,0001$ \\
Menos de 11 años de escolaridad & $1,53(1,29-1,80)$ & $<0,0001$ & $1,51(1,23-1,85)$ & $<0,0001$ \\
Gravidez 2 y más & $1,40(1,20-1,65)$ & $<0,0001$ & $3,55(2,70-4,68)$ & $<0,0001$ \\
Preeclampsia & $4,21(3,23-5,5)$ & $<0,0001$ & $2,89(1,62-5,18)$ & $<0,0001$ \\
Corioamnionitis & $1,87(1,13-3,10)$ & 0,0086 & &
\end{tabular}

CPN: control prenatal. 
La Tabla VIII muestra que tanto en el análisis bivariado como en el multivariado, la mala adherencia al CPN, la prematuridad y/o bajo peso al nacer y la carencia de seguridad social en salud, aumentan el riesgo de mortalidad neonatal.

El modelo CART (Figura 1) identificó que la carencia de seguridad social en salud, asociado a estado civil soltera, separa o viuda y una edad materna de 13-19 años son predictores de mala adherencia al CPN en $61,9 \%$; la carencia de seguridad social en salud, asociado a estado civil soltera, separa o viuda y una edad materna $\geq 20$ años, son predictores de mala adherencia al CPN en $81,8 \%$; mientras que tener seguridad social en salud, ser casada o vivir en unión libre y una escolaridad $\geq 11$ años, son predictores de buena adherencia al CPN en $83,7 \%$.

\section{Tabla VIII \\ IMPACTO DE LA MALA ADHERENCIA AL CONTROL PRENATAL AJUSTADO POR OTRAS COVARIABLES SOBRE LA MORTALIDAD NEONATAL}

\begin{tabular}{lcccc}
\hline & \multicolumn{3}{c}{ Mortalidad neonatal } \\
\cline { 2 - 5 } Determinantes & ORc (IC 95\%) & $\mathrm{p}$ & ORa (IC 95\%) & $\mathrm{p}$ \\
\hline Mala adherencia al CPN & $3,66(1,86-7,25)$ & $<0,0001$ & $2,81(1,47-5,38)$ & 0,0020 \\
Prematuridad y/o bajo peso al nacer & $5,62(2,59-13,5)$ & $<0,0001$ & $5,05(2,38-10,70)$ & $<0,0001$ \\
Carencia de seguridad social en salud & $3,57(1,31-8,38)$ & 0,0014 & $2,49(1,05-5,93)$ & 0,0390 \\
\hline
\end{tabular}

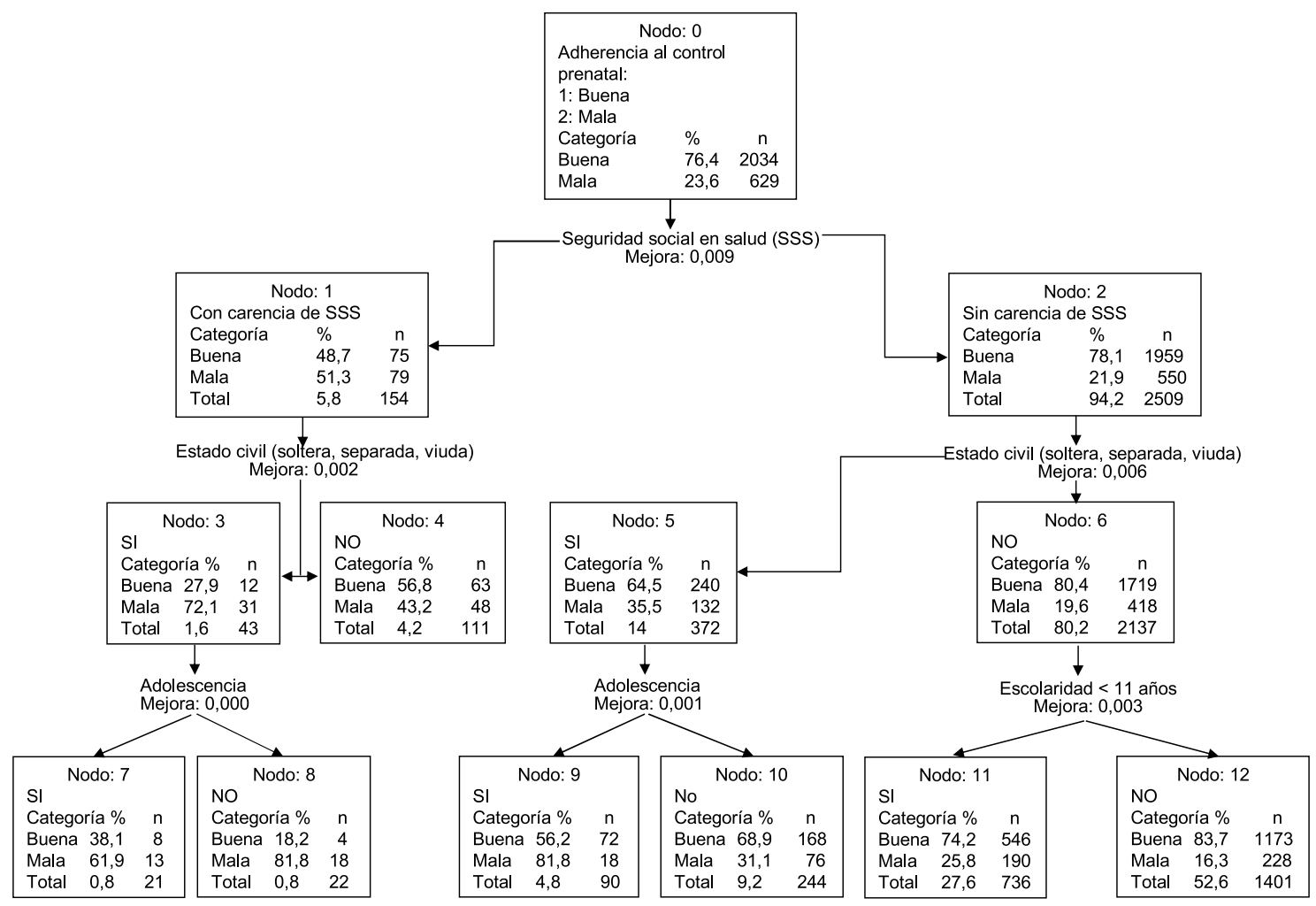

Figura 1. Modelo CART para adherencia al control prenatal ( $n: 2.663)$. 


\section{DISCUSIÓN}

El CPN reduce el riesgo de mortalidad del binomio madre-hijo siempre y cuando sea temprano, continuo, completo, extenso de cobertura y calidad. La salud guarda estrecha relación con múltiples determinantes sociales, culturales, genéticos, religiosos, demográficos, edad, nivel educativo, económico y ambiental (19). En este trabajo nos propusimos evaluar la influencia de la adolescencia y otras covariables sobre la adherencia al CPN y determinar el impacto de la mala adherencia al CPN ajustada por otras covariables, sobre el nacimiento prematuro y/o con bajo peso al nacer, y la mortalidad neonatal.

Encontramos que $21,4 \%$ de todas las madres cuyos hijos fueron hospitalizados eran adolescentes, cifra que se correlaciona con la informada en la literatura en la población general $(8,20)$, donde una de cada cinco adolescentes entre 15-19 años ha estado embarazada, sin embargo, es inferior a la informada para África Sub-Sahara, Oriente Medio y África del Norte, Este y Sur de Asia y El Pacífico, superior a las tasas de algunos países de Europa como República Checa, Hungría, Irlanda, Portugal, Gram Bretaña entre otros, así como superior a la informada para Estados Unidos de Norte América y Canadá, siendo la cifra similar para algunos países de Latino América como México, Nicaragua, Bolivia, Chile, Ecuador y Brasil (21-23) e inferior que en Ecuador (24).

Nuestros hallazgos identifican a las mujeres adolescentes como un grupo expuesto a inequidades en educación y en seguridad social en salud, tal como ha sido descrito por otros autores (25-27); y son estas barreras entre otras, las que hacen que las gestantes adolescentes no hagan un adecuado control prenatal o definitivamente no asistan. Nuestro estudio identificó que nuestras adolescentes gestantes ingresan más tardíamente al CPN, teniendo a su vez una menor número de visitas. Encontramos que 1 de cada 3 adolescentes y 1 de cada 5 madres $\geq 20$ años, no se adhirieron al CPN. A pesar de esto, las adolescentes tuvieron una significativa menor proporción de preeclampsia, pero una mayor proporción de nacimientos por cesárea y de corioamnionitis. De Vienne y cols (28), encontraron una significativa menor proporción de preeclampsia y de cesáreas entre las gestantes adolescentes, teniendo la adolescencia un efecto protector para la preeclampsia y para el nacimiento por cesárea.

Nuestros hallazgo identificaron a la adolescente como una población poco o mal adherente al CPN, con cifras de adherencia similares a la informada por otros autores, para quienes la adherencia al CPN está por debajo de $40 \%$ (29). La adherencia de este grupo al CPN es muy importante, debido al manejo que se puede ofrecer de manera oportuna a los múltiples factores de riesgo biológicos, psicológicos y socioculturales, que afectan a la adolescente gestante. Identificamos que la adolescencia es un determinante importante, pero no el único para una mala adherencia al CPN. EI CPN de las madres adolescentes ha sido materia de múltiples estudios, reportándose en este grupo un inicio tardío y una baja adherencia al mismo, en comparación con controles de gestantes adultas. Vieira y cols (26), describen que $43,7 \%$ (IC 95\%: 40-47,2\%) de adolescentes de $10-14$ años y $51,6 \%$ (IC 95\%: $50,8-52,4 \%$ ) de $15-$ 19 años tienen CPN adecuado. Un estudio realizado en Colombia, halló que $58,2 \%$ no asistieron a CPN, mientras que en las que asistieron $25,7 \%$ lo hicieron a uno o dos controles, $63,9 \%$ a tres a seis controles y $10,4 \%$ a más de seis controles (30). Ryan y cols (27), informan que la probabilidad de asistir al CPN es significativamente menor entre adolescentes de 10-14 años, pero no entre las gestantes de 15-19 años y mujeres adultas.

Son varias las barreras que experimentan las adolescentes para asistir al CPN, entre las cuales tenemos los problemas psicosociales, las barreras estructurales y las barreras actitudinales. Entre los facilitadores de la adherencia al CPN se describen el deseo de aprender a proteger su salud, la recepción de incentivos, la obtención de ayuda con el transporte y cuidado de sus otros niños (31). Otras barreras descritas afectan a los proveedores de salud (instituciones, médicos, enfermeras, etc.) encargados de atender al grupo de adolescentes, como acceso limitado a la información, actitudes negativas hacia los cambios en la práctica, falta de habilidades y deficiencia de recursos como acceso limitado a internet y educación. La propia formación médica intervencionista donde la preparación en la atención básica o de nivel I no es la adecuada o suficiente. Las instituciones de la salud que prestan estos servicios tienen escasez de personal profesional capacitado, de materiales necesarios, e instalaciones físicas no adecuadas para atender a la población adolescente embarazada (32).

Nuestros hallazgos muestran la asociación que hay entre una mala adherencia al CPN (entre otros determinantes) y resultados adversos como el nacimiento prematuro y/o con bajo peso al nacer y mortalidad neonatal. La adherencia al CPN es muy importante en la reducción del parto prematuro y bajo peso al nacer, como ha sido mostrado por trabajos realizados en Australia y Sudáfrica en los cuales se identificó que el CPN dirigido a esta población, es una intervención que disminuye estos eventos (29).

La adolescencia ha sido descrita como un determinante importante de prematuridad, bajo peso al nacer para la edad gestacional y bajo peso al nacer por diferentes autores $(12,33,34)$. Debiec y cols (35), encontraron que la no realización de CPN aumentaba el riesgo de parto pretérmino (ORa: 7,4 ; IC 95\%: 5,7-9,7), mientras que el realizar un 
CPN no adecuado (menor al $75 \%$ de lo esperado) aumentaba el riesgo de parto pretérmino entre un 30\%-150\% (IC 95\%: 10\%-230\%). A pesar que nosotros no hallamos una asociación directa entre adolescencia y riesgo de mortalidad neonatal, sabemos que este grupo fue menos adherente al CPN, y tal como lo mostraron Donoso y cols (36), Salihu y cols (37) y Mendoza y cols (38), el embarazo en la adolescencia, en especial antes de los 15 años de edad, se halla asociado a muerte fetal y neonatal. Otros autores han encontrado un aumento significativo de la mortalidad neonatal en madres menores de 18 años (39).

\section{CONCLUSIONES}

Esta investigación mostró que la edad materna de mayor riesgo para una mala adherencia al CPN es la adolescencia. Sin embargo, un hallazgo importante de este estudio, es que no sólo la edad se halló asociado a mala adherencia al CPN, sino que se destaca que otros factores del entorno materno están vinculados como, escolaridad materna, carencia de esposo o compañero permanente, carencia de seguridad social y número de embarazos. También se pudo evaluar el impacto negativo que tiene la mala adherencia al CPN y otros determinantes sobre el momento del parto, el cual puede darse antes del término en aquellas gestantes mal adherentes al CPN, como también el impacto negativo sobre el peso al nacer y la mortalidad neonatal. El sistema de salud debe constituirse en un facilitador que acompañe desde muy temprano a las adolescentes, favoreciendo un vínculo de confianza y respeto. En una futura investigación, se deben identificar barreras y facilitadores que determinen el ingreso oportuno y la permanencia de las adolescentes en embarazo en los CPN.

\section{REFERENCIAS}

1. División de Población de las Naciones Unidas, Panorama de la Población Mundial: Revisión 2006. Base de datos de población, 2006. Acceso: 5 de Enero de 2014. Disponible en: http://www.unicef.org/lac/serie_ pol.pulADOLESCENTES_ESP(2).pdf.

2. Organización Panamericana de Salud, $48^{\circ} \mathrm{CONSE}$ JO DIRECTIVO. 60.a SESIÓN DEL COMITÉ REGIONAL. Washington, D.C., EUA, del 29 de septiembre al 3 de octubre del 2008. Estrategia regional para mejorar la Salud de Adolescentes y Jóvenes, CE142/13 Rev. 2, 2008. Acceso: 7 de Enero de 2014. Disponible en: http://www.adolescenciaalape.org/sites/www.adolescenciaalape.org/files/estrateg_regional_para_mejorar_la_salud_de_los_adolesc_2008.pdf.

3. Lee MC, Suhng LA, Lu TH, Chou MC. Association of parental characteristics with adverse outcomes of adolescent pregnancy. Fam Pract 1998;15:336-42.

4. Klima CS. Centering pregnancy: a model for pregnant adolescents. J Midwifery Womens Health 2003;48:220-5
5. Parada A, Becerra D, Villacis C. Obstetricia Integral Siglo XXI: Embarazo en la Adolescencia. Universidad Nacional de Colombia, 2009.

6. Conde-Agudelo A, Belizán J, Lammers C. Maternalperinatal morbidity and mor $\rightarrow$ tality associated with adolescent pregnancy in Latin America: Cross-sectional study. Am J Obstet Gynecol 2005;192:342-9.

7. Organización Panamericana de Salud, Salud en las Américas, 2007. Acceso: 15 de Octubre de 2014. Disponible en: http://bvs.per.paho.org/videosdigitales/ matedu/RegionAmericas_fgonzales.pdf.

8. Plan Andino para la prevención del embarazo en adolescentes. Colombia. [1 pantalla]. Acceso: 1 de Noviembre de 2014. Disponible en: http://www.planandinopea. $\mathrm{org} / ? \mathrm{q}=$ node/60.

9. United States Agency for International Development, Profamilia. Salud sexual y reproductiva en zonas marginadas: situación de las mujeres desplazadas 2005. Bogotá: United States Agency International Development, Profamilia; 2005. Acceso: 1 de Octubre de 2014. Disponible en: http://www.unicef.org/lac/lNVERSION EN ADOLESCENTES(3).pdf.

10. Holguin YP, Mendoza LA, Esquivel CM, Sánchez R, Daraviña $A F$, Acuña $M$. Factores asociados al inicio de la actividad sexual en adolescentes de Tuluá, Colombia. Rev Chil Obstet Ginecol 2013; 78(3): 209-19.

11. World Health Organization. Adolescent pregnancy - Unmet needs and undone deeds. A review of the literature and programmes. Geneva: World Health Organization; 2007.

12. Chen XK, Wen SW, Fleming N, Demissie K, Rhoads GG, Walker M. Teenage pregnancy and adverse birth outcomes: a large population based retrospective cohort study. Int J Epidemiol 2007;36(2):368-73.

13. Hidalgo LA, Chedraui PA, Chavez MJ. Obstetrical and neonatal outcome in young adolescents of low socioeconomic status: a case control study. Arch Gynecol Obstet 2005;271(3):207-11.

14. Goldenberg P, Figueiredo Mdo C, Silva Rde S. Adolescent pregnancy, prenatal care, and perinatal outcomes in Montes Claros, Minas Gerais, Brazil. Cad Saude Publica 2005;21(4):1077-86.

15. Menacker FM, Martin JA, MacDorman MF, Ventura SJ. Births to 10-14 year-old mothers, 1990-2002: Trends and health outcomes: National Vital Statistics Reports - Centers for Disease Control and Prevention; 2004.

16. Parra M. Obstetricia Siglo XXI: Control Prenatal. Bogotá: Universidad Nacional de Colombia; 2009.

17. Jubiz A, Fuentes J. Atención prenatal. En: Botero J. Obstetricia y Ginecología. 7aㅡ Edición. Bogotá: CIB. 2004:108-17.

18. Galvis V, Bustamante MA, y Sarmiento CA. Norma técnica para la detección temprana de las alteraciones del embarazo. Colombia. Ministerio de Salud. Acceso: 20 de Octubre de 2014. Disponible en: http:// www.nacer.udea.edu.co/pdf/capacitaciones/hc/08deteccion.pdf.

19. Gallego LI, Vélez GA, Agudelo B. Panorama de la mortalidad materna. Colombia 2008;43(5 Pt 2):17816.

20. Profamilia, Ministerio de la Protección Social República de Colombia, Bienestar Familiar y United States Agency International Development, USAID del Pueblo de los Estados Unidos. Conocimientos del VIH y 
otras ETS, Encuesta Nacional de Demografía y Salud ENDS 2010. Informe de prensa, 2010. Acceso: 16 de Mayo de 2015. Disponible en: http://www.profamilia.org.co/encuestas/Profamilia/Profamilia/index. php?option=com_content\&view=article\&id=62\&ltem id $=9$.

21. Department of Child and Adolescent Health and Development. Department of Reproductive Health and Research. World Health Organization, Geneva. Adolescent Pregnancy. Issues in Adolescent Health and Development. 2004. Acceso: 16 de Mayo de 2015. Disponible en: http://whqlibdoc.who.int/publications/2004/9241591455_eng.pdf.

22. Plan Andino para la prevención del embarazo en adolescentes. Bolivia. [1 pantalla]. Acceso: 16 de Mayo de 2015. Disponible en: http://www.planandinopea. org/?q=node/58.

23. Plan Andino para la prevención del embarazo en adolescentes. Chile. [2 pantallas]. Acceso: 16 de Mayo de 2015. Disponible en: http://www.planandinopea. org/?q=node/59.

24. Plan Andino para la prevención del embarazo en adolescentes. Ecuador. [2 pantallas]. Acceso: 16 de Mayo de 2015. Disponible en: http://www.planandinopea.org/?q=node/61.

25. Sánchez YA, Mendoza LA, Grisales MB, Ceballos LY, Bustamante JC, Muriel E, Chaverra LA, Acuña ME. Características poblacionales y factores asociados a embarazo en mujeres adolescentes de Tuluá, Colombia. Rev Chil Obstet Ginecol 2013;78(4):26981.

26. Vieira CL, Coeli CM, Pinheiro RS, Brandao ER, Camargo Jr KR, Aguiar FP. Modifying effect of prenatal care on the association between young maternal age and adverse birth outcomes. J Pediatr Adolesc Gynecol 2012;25:185e189.

27. Ryan JA, Casapía M, Aguilar E, Silva H, Joseph SA, Gyorkos ThW. Comparison of prenatal care coverage in early adolescents, late adolescents, and adult pregnant women in the Peruvian Amazon. Int J Gynecol Obstet 2009;107:162-5.

28. de Vienne CM, Creveuil Ch, Dreyfus M. Does young maternal age increase the risk of adverse obstetric, fetal and neonatal outcomes: A cohort study. Eur J Obstet Gynecol Reprod Biol. 2009;147:151-6.

29. Quinlivan JA, Evans SF. Teenage antenatal clinics may reduce the rate of preterm birth: a prospective study. BJOG 2004;111(6):571-8.

30. Pardo F, Uriza G. Estudio de embarazo en adolescentes en 11 instituciones Colombianas. Rev Col Obstet Ginecol 1991;42(2):109-21.

31. Heaman MI, Moffatt M, Elliott L, Sword W, Helewa $\mathrm{ME}$, Morris $\mathrm{H}$, et al. Barriers, motivators and facilitators related to prenatal care utilization among inner-city women in Winnipeg, Canada: a case-control study. BMC Pregnancy Childbirth 2014;14:227.

32. Belizán M, Meier A, Althabe F, Codazzi A, Colomar $\mathrm{M}$, Bueken $\mathrm{P}$, et al. Facilitators and barriers to adoption of evidence-based perinatal care in Latin American hospitals: a qualitative study. Health Educ Res. 2007;22(6):839-53.

33. Fraser AM, Brockert JE, Ward RH. Association of young maternal age with adverse reproductive outcomes. N Engl J Med 1995;332(17):1113-7.

34. Malabarey OT, Balayla J, Klam SL, Shrim A, Abenhaim HA. Pregnancies in young adolescent mothers: a population-based study on 37 million births. J Pediatr Adolesc Gynecol 2012;25(2):98-102.

35. Debiec K.E., Paul K.J., Mitchell C.M., and Hitti J.E. Inadequate prenatal care and risk of preterm delivery among adolescents: a retrospective study over 10 years. Am J Obstet Gynecol 2010;203:122.e1-6.

36. Donoso E, Carvajal JA, Vera C, Poblete JA. La edad de la mujer como factor de riesgo de mortalidad materna, fetal, neonatal e infantil. Rev Med Chile 2014;142:168-174.

37. Salihu HM, Sharma PP, Ekundayo OJ, Kristensen S, Badewa AP, Kirby RS, et al. Childhood pregnancy (10-14 years old) and risk of stillbirth in singletons and twins. J Pediatr 2006;148(4):522-6.

38. Mendoza LA, Arias MD, Mendoza LI. Hijo de madre adolescente: riesgos, morbilidad y mortalidad neonatal. Rev Chil Obstet Ginecol 2012;77(5):375-82.

39. Olausson PO, Cnattingius S, Haglund B. Teenage pregnancies and risk of late fetal death and infant mortality. Br J Obstet Gynaecol 1999;106 (2):116-21. 